\title{
MOBILE MEDICAL CAMP REPORT
}

Rana SJB*, Neopane A*, Subba K*

A mobile medical camp organized by RNAMC was setup in No. 4 Brigade HQ. Nimare Barrack, Surkhet. The duration of the camp was of six days i.e. from $15^{\text {th }}$ Mangshir 2061 to $21^{\text {st }}$ Mangshir 2061.

The 34 strength mobile medical team members were as follows:

\section{Brig Gen Dr Suraj SJB Rana Team Leader Ophthalmologist}

2. Col Dr Gobinda Khadka Radiologist

3. Col Dr Arya Pandey Obstetrician \& Gynaecologist

4. Lt Col Dr Naresh Giri Surgeon

5. Li Col Dr Arun Neopane Pediatrician

6. Lt Col Dr Nagendra Prasad Shah Oto-Rhino-Laryngologist

7. Lt Col Dr Bharat Prasad Singh Orthopedic Surgeon

8. Lt Col Dr Arun Sharma Physician

9. Maj Dr Uday Bajracharya Anesthesiologist

10. Capt Dr Nirjala Aryal GDMO

11. Lt Dr Riwaj Karki Dental Surgeon

12. Lt Dr Poonam Chand Thakuri GDMO

13. Lt Col Kamala Subba Specialised Nurses

14. Capt Sarala Thapa Staff Nurse

* Dr. Suraj SJB Rana Brig. Gen., Team Leader Consultant Ophthalmologist

* Dr. Arun Neopane Lt. Col., Pediatrician

* Mrs. Kamala Subba Lt. Col., Nursing Officer
15. Lt Bina Darai Staff Nurse

16. T/Sub Sarala Sapkota Staff Nurse

17. T/Capt Khagendra Si Ma Medical Lab Technologist (Adam. Officer)

18. T/Capt. Tilak Bahadur Khadka O.T. Technician (Logistic Officer)

19. T/Sub Nakul Thapa Paramedical Staff - (Ophthalmology)

20. T/Sub Sanu Maiya Khadka Paramedical Staff - (Obst./Gynae)

21. T/Jem Guman Singh Pu Ma Paramedical Staff - (Anesthesiology)

22. T/Sub Ram Narayan Paudyal Paramedical Staff - (Dispensary)

23. T/Jem Yem Bdr Godar Paramedical Staff - (Surgery)

24. T/Jem Yagya Purush Pandey Paramedical Staff - (Radiology)

25. T/Hav Shankar Poudel Paramedical Staff

26. T/Hav Ramesh Thapa Paramedical Staff

27. T/Hav. Maheshwor Chaudhary Paramedical Staff

28. T/Hav Gir Bdr Thapa (DMI) Photography

29. T/Hav Ramji Prasad Devkota Paramedical Staff

30. T/Hav Jaman Singh Basnet Paramedical Staff

31. T/Nk. Ananda Bhandari Paramedical Staff

32. T/Nk Mohan Thapa Paramedical Staff

33. T/Nk Dhan Bdr Rai Paramedical Staff

34. Sep. Lok Raj Parajulee Paramedical Staff 
Specialised medical services were provided in the following fields. General Medicine, General Surgery, Obstetric \& Gynaecology, Pediatric, Orthopedic, Anesthesia, Ophthalmology, Otorhinolaryngology, Radiology, Pathology and Dental. The task given to the team was to provide medical service to the serving soldiers their families and the lix. servicemen and their families cover by the $4^{\text {th }}$ Brigade. The expected patient load was approximately 1750 (i.e. Ex-servicemen and families) and 200 regular soldiers. The Kalyankari branch through the DMS office provided the medicines. The instruments and equipments were taken from Shree Birendra Hospital. It took three trips for the "Sky Truck" to transport the team members \& the equipments to Surkhet. The first two trips were flown on 14th of Mangshir and the last trip came on the 15th. The team started work right from the 15th of Mangshir in spite of the incomplete manpower.

\section{Procedures}

\begin{tabular}{|l|l|l|c|c}
\hline S.No. & Department & Procedurc & No. of Cases & Remarl \\
\hline 1. & ENT & Uvelectomy & 1 & \\
\hline & & Inferior Turbinectomy & 1 & \\
\hline 2. & Foreign body removal nose (Insect) & 2 & \\
\hline & & Excision of Papiloma soft palate & 1 & \\
\hline 3. & Eye & Extractions & 49 & \\
\hline & & Fillings & 113 & \\
\hline & & Refraction & 263 & \\
\hline & & FEUM & 42 & \\
\hline & & Syringing & 65 & \\
\hline & & Schiotz tonometry & 46 & \\
\hline & & Schirmers test & 135 & \\
\hline 4. & Obst./Gynae & D \& C : For incomplete abortion & 1 & \\
\hline & & Laprotomy and excision of Twisted & & \\
\hline & & ovarian cyst (Emergency) & 1 & \\
\hline 6. & Radiology & Chest X-Rays & 61 & \\
\hline & & LS/Cx Spine & 120 & \\
\hline & & & & \\
\hline
\end{tabular}

The camp was setup in the previous $N_{0}{ }_{4}$ ambulance complex. The space available $w_{a}$ sufficient however we managed to squeeze it specialised OPDs, the Pathology Labor" Dispensary, X-Ray, USG, ECG and Refra to an O.T. with pre and post-operative and $C$ facilities. There were two beds for pre-oper patients and four beds for post-operative pati The pre-operative beds were also used f, patients.

Patient Registration was a tough job at first but on it was more organized. Daily about three of the patients who reported to the Arti Battalion in Mangalgarhi Barrack situatc Surkhet bazaar, 7 kilometers away from Bri HQ were brought by bus to the camp location. I patients however came from the near by Bri HQ area. The No 4 Brigade HQ staffs provider support. They provided the necessary sect administration support, cooperation and the $v$ hospitality. units. The Brigade MI room, which was conve 


\begin{tabular}{|l|l|l|l|l}
\hline & & Joint/Bone & 98 & \\
\hline & & KUB & 31 & \\
\hline & & PNS & 4 & \\
\hline & Skull & 1 & \\
\hline & & USG Abdo & 170 & \\
\hline & Pathology & USG Thyroid & 169 & \\
\hline & & Stool RE & 108 & \\
\hline & & Pregnancy tests & 47 & \\
\hline & & HIV test & 48 & \\
\hline & & Blood RE & 125 & \\
\hline & & LFT & 67 & \\
\hline & & Blood grouping & 72 & \\
\hline & & Semen analysis & 2 & \\
\hline & & Renal function test & 138 & \\
\hline & & Blood Sugar test & 101 & \\
\hline & & Uric Acid & 90 & \\
\hline & & Hbs Ag & & \\
\hline
\end{tabular}

Following Surgeries were performed at Surkhet Medical Camp

Surgeon: Lt. Col. Dr. N.K. GiriAnaesthesiologist: Maj. Dr. U.B. Bajracharya

\begin{tabular}{|c|c|c|c|c|c|c|c|}
\hline S.No. & Rank & Name of Patient & Age & Sex & Diagnosis & Operation & Anacsthesia \\
\hline \multicolumn{8}{|c|}{ Date: $2061 / 8 / 15$ (Day 1) } \\
\hline 1. & Faumily & $\begin{array}{l}\text { Mr. Surendra Basyal } \\
\text { S/O Hav Arjun Basyal }\end{array}$ & $10 \mathrm{yrs}$ & M & $\begin{array}{l}\text { Small lump abd and } \\
\text { mass ring finger }\end{array}$ & Excicion & LA \\
\hline 2. & Family & $\begin{array}{l}\text { Mr. Arjun Basyal } \\
\text { S/O Hav. Arjun Basyal }\end{array}$ & $9 \mathrm{yrs}$ & M & Mole LA. leg & Excicion & LA \\
\hline 3. & Family & $\begin{array}{l}\text { Mr. Suman K. Pariyar } \\
\text { S/O Nk. Shankar Pariyar }\end{array}$ & 8 yrs & $M$ & Abscess Rt. ankle & $1 / \mathrm{D}$ & GA \\
\hline \multicolumn{8}{|c|}{ Date: 2061/8/16 (Day 2) } \\
\hline 1. & Family & Master Sushil Poudel & $2 \mathrm{yrs}$ & $\mathrm{M}$ & Phimosis & Circumcision & GA \\
\hline 2. & $\mathrm{Hav} / \mathrm{Clk}$ & Bhakta Bdr Tamang & $28 \mathrm{yrs}$ & $\mathrm{M}$ & Anal tag & Excission & LA \\
\hline 3. & Lnk. & Krishna Bdr Ra.Ma. & $25 \mathrm{yrs}$ & $M$ & Auricular cyst & Aspiration & LA \\
\hline 4. & Family & Master Kiran Achhami & $3 \mathrm{yrs}$ & M & Phimosis & Circumcision & GA \\
\hline 5. & $\mathrm{Nk}$. & Yam Bdr Oli & $25 \mathrm{yrs}$ & $\mathrm{M}$ & Ingrowing toe nail & Excission & LA \\
\hline 6. & Nk. & Sher Bdr Basnet & $33 \mathrm{yrs}$ & $M$ & Epigastric hernia & Hernia repair & LA \\
\hline 7. & $\mathrm{Nk}$ & Bharaı Bi.Ka. & $38 \mathrm{yrs}$ & M & Lipoma & Excission & LA \\
\hline 8. & Family & Mr. Ganga Bdr Shahi & $11 \mathrm{yrs}$ & $M$ & Cervical lymphadinitis & Excission & LA \\
\hline
\end{tabular}




\begin{tabular}{|c|c|c|c|c|c|c|c|}
\hline 9. & Hav. & Arjun Ranabhat & 28 yrs & M & Multiple lipoma & Excission & \\
\hline 10. & Hav. & Bhim Bdr Sherpa & 32 yrs & $\mathrm{M}$ & Multiple lipoma & Excission & \\
\hline 11. & Family & Mr. Hikmat Sherman & $10 \mathrm{yrs}$ & M & Ganglion Rt. hand & Excission & \\
\hline 12. & Family & Miss Tika Bi.Ka. & 7 yrs & $\mathrm{F}$ & Wart & Excission & \\
\hline 13. & Family & Mrs. Laxmi Kafle & 25 yrs & $\mathrm{F}$ & Foreign body Rt. hand & Excission & \\
\hline 14. & Family & Mrs. Parbati Bi.Ka. & 53 yrs & $\mathrm{F}$ & Calcinosis cutis Rt. hip & Excission & LA \\
\hline 15. & Family & Mr. Raju Darlami & $18 \mathrm{yrs}$ & $\mathrm{M}$ & $\begin{array}{l}\text { Incised wound Lt. } \\
\text { index finger }\end{array}$ & Suturing & $\widehat{L A}$ \\
\hline \multicolumn{8}{|c|}{ Date: 2061/8/17 (Day 3) } \\
\hline 1. & Sep. & Jit Bdr Shrestha & $22 \mathrm{yrs}$ & $\mathrm{M}$ & Hematoma anal region & Excission & $\widehat{S A}$ \\
\hline 2. & Family & Mr. Laxchay Magar & $5 \mathrm{yrs}$ & $\mathrm{M}$ & \begin{tabular}{|l|} 
Hydrocele \\
\end{tabular} & Hydrocelectomy & $\overline{\mathrm{GA}}$ \\
\hline 3. & Family & Mris Purna Lamichhane & $14 \mathrm{yrs}$ & $\mathrm{F}$ & Sebacious cyst scalp & Excission & LA \\
\hline 4. & Family & Mrs. Rasila Baduwal & $23 \mathrm{yrs}$ & $\mathrm{F}$ & Appendicular lump & Appendicectomy & SA \\
\hline 5. & Family & Mr. Danda Lal Regmi & $57 \mathrm{yrs}$ & $\mathrm{M}$ & Lt. inguinal Hernia & Hernioraphy & $\widehat{S A}$ \\
\hline 6. & Family & Mr. Naresh Bista & $14 \mathrm{yrs}$ & $\mathrm{M}$ & Cyst scalp & Excission & LA \\
\hline 7. & Sep. & Chitra Bdr Bu.Chhe. & $21 \mathrm{yrs}$ & $\mathrm{M}$ & Hydrocele Ll. & Hydrocelectomy & LA \\
\hline 8. & Family & Mrs. Basundhara Acharya & $55 \mathrm{yrs}$ & $\mathrm{F}$ & Scbacious cyst & Excission & LA \\
\hline 9. & Sep. & Mohan Thapa & $20 \mathrm{yrs}$ & $M$ & Lipoma & Excission & LA \\
\hline 10. & Family & Master Pawan Thapa & 18 months & $\mathrm{M}$ & Inguinal abscess & $I \& D$ & $\mathrm{G} t$ \\
\hline 11. & Sep. & Khagendra Katuwal & $24 \mathrm{yrs}$ & $\mathrm{M}$ & Sebacious cyst & Excission & $\mathrm{L} t$ \\
\hline 12. & Family & Mr. Pradeep Ghale & $5 \mathrm{yrs}$ & $\mathrm{M}$ & Congenital hydrocele & Herniotomy & $\mathrm{G} t$ \\
\hline 13. & Family & Mrs. Hira Maya Bi.Ka. & $28 y$ ts & IF & Ganglion Lt. wrist & Excission & $\mathrm{LA}$ \\
\hline 14. & Family & Mr Decpak Khadka & $10 \mathrm{yrs}$ & $\mathrm{M}$ & Congenital hydrocele & Herniotomy & $\mathrm{G} t$ \\
\hline 15 & Family & Mr. Tek Bdr Rana & $19 \mathrm{yrs}$ & $\mathrm{M}$ & Lipoma back & Excission & $\mathrm{L} A$ \\
\hline 16. & Family & Mr. Subinil Chandra & $4 \mathrm{yrs}$ & $\mathrm{M}$ & Sebacious cyst scalp & Excission & $\mathrm{L} f$ \\
\hline 17. & Family & Mrs. Bhagwoti chand & $24 \mathrm{yrs}$ & $\mathrm{F}$ & Incomplete abortion & $D \& C$ & $\overline{\mathrm{G} t}$ \\
\hline 18. & Sep. & Chok Bdr Khadka & $24 \mathrm{yrs}$ & $\mathrm{M}$ & Family planning & Vasectomy & $\mathrm{L} f$ \\
\hline 19. & Family & Mr. Mohan Bi.Ka. & 4 yrs & $\mathrm{M}$ & Tongue tie & Release & $\mathrm{G} /$ \\
\hline 20. & Family & Mr. Jap Bdr Budha & $69 \mathrm{yrs}$ & $\mathrm{M}$ & Lipoma scalp & Excission & $\mathrm{L} t$ \\
\hline \multicolumn{8}{|c|}{ Date: $2061 / 8 / 18$ (Day 4) } \\
\hline 1. & Family & Master Suman Thapa & $2 \mathrm{yrs}$ & $M$ & Phimosis & Circumcision & $\mathrm{G}_{2}$ \\
\hline 2. & Family & Mr. Swapnil Chand & $4 \mathrm{yrs}$ & $\mathrm{M}$ & Phimosis & Circumcision & $\mathrm{G}_{2}$ \\
\hline 3. & Family & Mr. Decpak Khadka & $10 \mathrm{yrs}$ & $\mathrm{M}$ & Congenital hydrocele & Herniotomy & $\mathrm{G}_{i}$ \\
\hline 4. & Family & Mr. Pradeep Ghale & 5 yrs & $\mathrm{F}$ & Congenital hydrocele & Herniotomy & $\mathrm{G}_{i}$ \\
\hline 5. & Family & Mr. Tika Ram Pandey & $7 \mathrm{yrs}$ & $\mathrm{M}$ & Corn Rt. sole & Excission & $\mathrm{L} /$ \\
\hline 6. & Family & Mrs. Khagicwara Saru & $40 \mathrm{yrs}$ & $\mathrm{F}$ & Bursitis LI. knce & Excission & $\mathrm{L} /$ \\
\hline 7. & Family & Miss Elisha Singh & $6 \mathrm{yrs}$ & $\mathrm{F}$ & Ingrowing toe nail & Excission & $\mathrm{L} /$ \\
\hline 8. & Family & Mrs. Usha Basnet & $21 \mathrm{yrs}$ & $\mathrm{F}$ & Fibroadenoma & Excission & $\underline{L}$ \\
\hline 9. & Family & Mrs. Maina Kandel & $25 \mathrm{yrs}$ & $F$ & Galactococle & Excission & G. \\
\hline 10. & Family & Mr. Mito Lal Bi.Ka. & $10 \mathrm{yrs}$ & $\mathrm{M}$ & Septic arthritis & Arthrotomy & G. \\
\hline 11. & Nk. & Khim Lal Bi.Ka. & $33 \mathrm{yrs}$ & $\mathrm{M}$ & Ganglion Rt. ankle & Excission & $\mathrm{L}$ \\
\hline 12. & Lnk. & Khadga Lal Basyal & $23 \mathrm{yrs}$ & $\mathrm{M}$ & Ingrowing toc nail & Excission & $L$ \\
\hline 13 & Lnk. & Botha Bdr Budhathoki & $24 \mathrm{yrs}$ & $\mathrm{M}$ & Lipoma & Excission & L \\
\hline
\end{tabular}




\begin{tabular}{|c|c|c|c|c|c|c|c|}
\hline 14. & Sep. & Mangal Kumal & $32 \mathrm{yrs}$ & $\mathrm{M}$ & Lipoma & Excission & LA \\
\hline 1.5 & WO2 & Jit Lal Lama & $35 \mathrm{yrs}$ & $\mathrm{M}$ & Corn Rt. sole & Excission & $\mathrm{LA}$ \\
\hline 16. & WO2 & Keshab Humagai & $35 \mathrm{yrs}$ & $M$ & Corn Rt. sole & Excission & LA \\
\hline \multicolumn{8}{|c|}{ Date: 2061/8/19 (Day 5) } \\
\hline 1. & Family & Mr. Kamal Gurung & $11 \mathrm{yrs}$ & $\mathrm{M}$ & Bladder Stone & Vesiculolithotomy & $\mathrm{GA}$ \\
\hline 2. & Family & Mr. Kamal Tha.Ma. & 8 yrs & $\mathrm{M}$ & Congenital hydrocele & Herniotomy & GA \\
\hline 3. & Fannily & Mr. Padam Rout & $14 \mathrm{yrs}$ & $\mathrm{M}$ & Gynaecomastia & Excission & GA \\
\hline 4. & Fannily & Mr. Som Bdr Saı.Ma. & $10 \mathrm{yrs}$ & $\mathrm{M}$ & Anal polyp & Excission & GA \\
\hline 5 & Family & Miss Dil Kumari Rout & $12 \mathrm{yrs}$ & $\mathrm{F}$ & Lipoma neck & Excission & GA \\
\hline 6. & Family & Miss Roshani Giri & $4 \mathrm{yrs}$ & $\mathrm{F}$ & Blecding $P / R$ & EUA & $\mathrm{GA}$ \\
\hline 7 & Lnk. & Kaji Ram Rout & $20 \mathrm{yrs}$ & $\mathrm{M}$ & Ingrowing toe nail & Excission & LA \\
\hline 8. & Family & Mr. Bhushan Hamal & $7 \mathrm{yrs}$ & $M$ & Congenital hydrocele & Herniotomy & $\mathrm{GA}$ \\
\hline 9. & Family & Mrs. Laxmi Shahi & $40 \mathrm{yrs}$ & $\mathrm{F}$ & Cervical Lymphadenitis & Excission biopsy & $\mathrm{LA}$ \\
\hline 10. & Family & Mrs. Bhagwoti Thapa & $25 \mathrm{yrs}$ & $F$ & Corn Rt. sole & Excission & LA \\
\hline 11. & Family & Mrs. Golma Thapa & $50 \mathrm{yrs}$ & $\bar{F}$ & Lipoma Rt. clbow & Excission & $\mathrm{LA}$ \\
\hline 12. & Ri. & Mahendra Bdr Shahi & $20 \mathrm{yrs}$ & $M$ & Forcign body Rt. hand & Excission & LA \\
\hline 13. & Sub. & Netra Bdr Khattri & $40 \mathrm{yrs}$ & $\mathrm{M}$ & Lipoma Rt. hand & Excission & LA \\
\hline 14. & Sep. & Rabindra Rai & $20 \mathrm{yrs}$ & $\mathrm{M}$ & $\begin{array}{l}\text { Small nodule } \\
\text { RL. middle finger }\end{array}$ & Excission & $\mathrm{LA}$ \\
\hline \multicolumn{8}{|c|}{ Date: 2061/8/20 (Day 6) } \\
\hline 1 & Family & Mr. Ishanta Oli & $4 \mathrm{yrs}$ & $\mathrm{M}$ & Congenital hydrocele & Herniotomy & $\mathrm{GA}$ \\
\hline 2. & Family & Mr. Deepak Wagle & $11 \mathrm{yrs}$ & $\mathrm{M}$ & Congenital hydrocele & Herniotomy & $\mathrm{GA}$ \\
\hline 3. & Famuly & Mr. Bhupemdra Acharya & $21 \mathrm{yrs}$ & $\mathrm{M}$ & Congenital hydrocele & Herniotomy & $\mathrm{GA}$ \\
\hline 4. & Family & Mrs. Mecna Chand & $33 \mathrm{yrs}$ & $\mathrm{F}$ & Twisted ovarian cyst & Laparotomy & $\mathrm{GA}$ \\
\hline 5. & Police & Prem Bdr Budhathoki & $25 \mathrm{yrs}$ & $\mathrm{M}$ & Post auricular cyst & Excission & $\mathrm{LA}$ \\
\hline 6 & Lnk. & Narayan Bhandari & $35 \mathrm{yrs}$ & $M$ & Wart penis & $\begin{array}{l}\text { Diathermy } \\
\text { Excission }\end{array}$ & $\mathrm{LA}$ \\
\hline 7. & Nk. & Buda Bdr. Bu.Chhe. & $33 \mathrm{yrs}$ & $\mathrm{M}$ & Lipoma Rt. hand & Excission & LA \\
\hline 8. & Maj. & Harka Bdr Shahi & $36 \mathrm{yrs}$ & $M$ & Skin tag neck & $\begin{array}{l}\text { Diathermy } \\
\text { Excission }\end{array}$ & LA \\
\hline 9 & Family & Mrs. Chandra Kala Giri & 31 yrs & F & Corn Ri. sole & Excission & LA \\
\hline 10 & Sep. & Kamal Thapa & $23 \mathrm{yrs}$ & $M$ & Ganglion Lt. wrist & Excission & LA \\
\hline 11. & Family & Mr. Jagu Ram Tharu & $50 \mathrm{yrs}$ & $\mathrm{M}$ & Corn Rt. sole & Excission & LA \\
\hline 12 & Family & Mr. Ram Bdr Bohora & $50 \mathrm{yrs}$ & $M$ & Sebaceous cyst scalp & Excission & LA \\
\hline 13 & Formily & Mrs. Gopi Sara Gharti & $20 \mathrm{yrs}$ & $\mathrm{M}$ & Forcign body Ri. hand & Excission & LA \\
\hline 14. & Nk. & Dhan Bdr Shahi & $26 \mathrm{yrs}$ & $\mathrm{M}$ & Lump occipital region & Excission & $\mathrm{LA}$ \\
\hline \multicolumn{8}{|c|}{ Date: 2061/8/21 (Day 7) } \\
\hline$i$. & Sep. & Chet Naraya shrestha & $25 \mathrm{yrs}$ & $M$ & $\begin{array}{l}\text { Splinter injury } \\
\text { Lt. arm, Rt. Leg }\end{array}$ & Splinter removal & GA \\
\hline 2. & Lnk. & Gaja Bdr Ra.Ma. & $28 \mathrm{yrs}$ & $\mathrm{M}$ & $\begin{array}{l}\text { Splinter injury Lt. arm, } \\
\text { open \# Lt. femur, } \\
\text { laceration Lt. part of face }\end{array}$ & $\begin{array}{l}\text { Splinter removal, } \\
\text { Wound debridement }\end{array}$ & $\mathrm{GA}$ \\
\hline 3. & Lnk. & Givinda rana Magar & $25 \mathrm{yrs}$ & $\mathrm{M}$ & Splinter injury Rt. buttock & Splinter removal & LA \\
\hline
\end{tabular}









\section{Mobile Medacl Camp Surkhet}

\section{Histopathological Impression of Surgical Specimens}

\begin{tabular}{|c|c|c|c|c|}
\hline S.No. & Biopsy No & Name & Clinical Diagnosis & Histological Impression \\
\hline 1. & $22.3 \mathrm{R}$ & Nk. Khem Lal Bi.Ka. & $\begin{array}{l}\text { Calcified Ganglion } \\
\text { (Ankle) }\end{array}$ & Forcign Body Granuloma \\
\hline 2. & $224 \mathrm{R}$ & Hav. Arjun Ranabhat & Multiple Lipoma & Lipoma \\
\hline 3. & $225 R$ & Nk. Bharat B.K. & Multiple Lipoma & Lipoma \\
\hline 4. & $226 \mathrm{R}$ & Sub. Netra Bahadur Khatri & Lipoma & Lipoma \\
\hline 5. & $651 \mathrm{~F}$ & Meena Chand & Ovarian cyst (Rt.) & Mucinous Cystadenoma \\
\hline 6. & $652 \mathrm{~F}$ & Laxmi Shahi & Cervical Lymphnode & $\begin{array}{l}\text { Caseous Necrosis (AFB Negative) } \\
\text { - Consistent with Tuberculous } \\
\text { Lymphadenopathy }\end{array}$ \\
\hline 7. & $653 \mathrm{~F}$ & Som Bahadur Sa.Ma. & Anal Polyp & Juvenile (Retention) Polyp \\
\hline 8. & $654 \mathrm{~F}$ & Parbati Sunar & Skin Lump & Calcinosis Cutis \\
\hline 9. & $655 \mathrm{~F}$ & Usha Basnet & Breast Lump & Fibroadenoma (Intracanalicular) \\
\hline 10. & $656 \mathrm{~F}$ & Surendra Basyal & $\begin{array}{l}\text { Cyst - Anterior } \\
\text { Abdominal Wall }\end{array}$ & Cysticercus \\
\hline 11. & $657 \mathrm{~F}$ & Arjun Basyal & Mole & Hemangioma (Cavernous) \\
\hline 12. & $658 \mathrm{~F}$ & Gagan Shahi & Cervical Lymph node & $\begin{array}{l}\text { Reactive Para cortical Hyperplasia } \\
\text { (Reactive Lymphadenopathy) }\end{array}$ \\
\hline 13. & $659 \mathrm{~F}$ & Tek Bahadur Rana & Lipoma (Back) & Neurofibroma \\
\hline 14. & $660 \mathrm{~F}$ & Padam Raut & Breast Lump & Gynaccomastia \\
\hline 15. & $661 \mathrm{~F}$ & Purna Lamichhane & Hemangioma Scalp & Hemangioma \\
\hline 16. & $662 \mathrm{~F}$ & Rasila Baduwal & Appendix & $\begin{array}{l}\text { Early Inflammatory } \\
\text { Changes in Appendix }\end{array}$ \\
\hline 17. & $663 \mathrm{~F}$ & Mina Kandel & Breast Lump & Galactocele \\
\hline 18. & $664 \mathrm{~F}$ & Dil Kumari Raut & Hemangioma (Postauricular) & Angiomatosis \\
\hline 19. & $665 \mathrm{~F}$ & Khagisara Saru & Knee Swelling & Chronic Bursitis \\
\hline
\end{tabular}

\section{Conclusions}

1. The planning of such Mobile Medical Camp should be done well in advanced.

2. The selected team should have ample time to organize the camp.

3. Good coordination with No 11 Brigade and No 16 Brigade for air and land transport.

4. Portable light equipments should be used.

5. Portable dental chair with portable dental unit with dental air compressor.
6. This type of ambulatory surgery and anesthesia was first of its kind in the medical history of RNAMC.

7. For major surgeries, Mobile ICU (Air/Ground) should be arranged to transfer patient perioperatively if required.

8. Mobile blood bank will be an intelligent idea if it is feasible.

9. More GDMOs, female nurses and nursing assistants required.

10. OT, Surgical assistants/anesthesia assistants/ Scrub sisters were not enough. 
11. Ambulatory surgeries should be continued. Standard preparations should be uplifted. For fast tracking after ambulatory surgeries, short acting drugs like propofol, fentanyl, mivacurium, vecuronium, rocuronium, sevoflurance should be used.

12. A large number of cases were pediatric anesthesia and pediatric surgeries.

13. Boyle's Machine with OMV (Oxford Miniature vaporizer) would be a better choice. Tri service apparatus another option.

14. A portable OT table \& good OT lighting system are a must.

15. Better CSSD facilities should be provided.

16. There should be a system of identify the families of the both in-service soldiers and the Ex-servicemen.

17. The patients flow was tremendous and the team saw and treated more than 3300 patients (i.e. Ex-servicemen \& families totaling to about 2700 \& Regular Soldiers approximately $600)$.

18. Medicine which were taken from Kathmandu was meagerly enough for the camp duration but the estimated amount for the estimated patients was just enough except for a few drugs which were scarce. The dispensary managed to adjust a few medications in the later stages with some restrictions and reservations.

19. The various equipments taken by the respected departments were adequate but were not up to the field standards, which meant packing transporting them was a big problem. Medical equipments have to be procu such camps are to be conducted reg because the hospital equipments are bot get damaged.

20. The coordination part deserves some $\mathrm{mc}$ especially with the No 11 Brigade side,' was very poor. Flight scheduling, timin load calculations were not satisfac coordinated. The withdrawal plan was miscalculated also.

21. Coordination with the local commandes not very problematic as they did their best to help the medical corps conduc। camps, in spite of their hectic duties and responsibilities.

22. Medical side we found that the camp ' have been more effective if we had some specialties involved like the Ortho surgeon, Dermatologist, Pathologis maybe even a Pharmacologist to take c: medicine procurement and dispensing.

23. Such medical camps should be provided needy families and retired soldiers 0 : Royal Nepalese Army on a regular 1 Geographically deprived and crisis aff patients should get the priority, which n that the patients in the far west shoul more medical facilities than the economi geographically and medically privil patients in the east of our country. 